\title{
Characterization of nanoparticle transport through quartz and dolomite gravels by magnetic resonance imaging
}

\author{
S. Lakshmanan • W. M. Holmes • W. T. Sloan • \\ V. R. Phoenix
}

Received: 19 September 2014/Revised: 4 January 2015/Accepted: 27 January 2015/Published online: 17 February 2015

(C) Islamic Azad University (IAU) 2015

\begin{abstract}
Magnetic resonance imaging (MRI) has tremendous potential for revealing transport processes in engineered and geologic systems. Here, we utilize MRI to image nanoparticle (NP) transport through a saturated coarse-grained system. Commercially available paramagnetically tagged NPs are used; the paramagnetic tag making the NP visible to MRI. NP transport was imaged as NPs migrated through packed columns of quartz and dolomite gravel. Changes in T2-weighted image intensity were calibrated to provide fully quantitative maps of NP concentration at regular time intervals $\left(T_{2}\right.$ being the spin-spin relaxation time of ${ }^{1} \mathrm{H}$ nuclei). Transport of nanoparticles was significantly retarded in dolomite compared to quartz due to electrostatic attraction between nanoparticle and dolomite surfaces. NP concentration profiles were evaluated with the CXTFIT computer package to estimate nanoparticle transport parameters at multiple points along the length of the column. This provided temporally resolved parameters that standard breakthrough curve
\end{abstract}

S. Lakshmanan $(\bowtie) \cdot$ V. R. Phoenix

School of Geographical and Earth Sciences, University

of Glasgow, Gregory Building, Glasgow, UK

e-mail: susithra02@gmail.com

Present Address:

S. Lakshmanan

Department of Chemical Engineering and Biotechnology,

University of Cambridge, Cambridge, UK

W. M. Holmes

GEMRIC, Wellcome Surgical Institute, Institute

of Neuroscience and Psychology, University of Glasgow,

Glasgow, UK

W. T. Sloan

School of Engineering, University of Glasgow,

Rankine Building, Glasgow, UK analysis cannot provide. Particle-surface interaction energy profiles were described through Derjaguin-LandauVerwey-Overbeek (DLVO) theory. While dispersion coefficients and fast deposition rate constant $\left(k^{\text {fast }}\right)$ were found to increase with distance, deposition rate constant $(k)$ and collision efficiency $(\alpha)$ were found to decrease with distance. These length-dependant variations have significant scaling-up implications for transport models used to predict NP transport in natural and engineered coarsegrained systems, such as sustainable urban drainage systems and river beds.

Keywords Porous media $\cdot$ Nanoparticles $\cdot$ Magnetic resonance imaging - Transport - DLVO - Environment

\section{Introduction}

While the advantages afforded by nanotechnology are considerable and diverse, ecotoxicological studies have demonstrated that exposure of aquatic organisms to some nanoparticles can produce deleterious effects. These include vascular and respiratory injury, brain and liver damage and delayed embryonic development (Handy et al. 2008). Ecotoxicological studies have demonstrated that the aquatic environment is particularly at risk of exposure to nanoparticles (Scown et al. 2007). A range of ecotoxicological effects have also been reported, including effects on microbes, plants, invertebrates and fish (John H. Tellam 2011; Boxall et al. 2010). The potential to generate toxicity through the harmful interactions with biological systems and the environment is discussed by Nel et al. 2013. For many organisms, cytotoxicity and inflammatory responses lead to the loss of biodiversity and ecosystem functions ( $\mathrm{Nel}$ et al. 2006). Consequently, accurate data on 
nanoparticle (NP) transport in aquatic systems are needed to underpin the models used in environmental decisionmaking.

Recognizing the need for data on nanoparticle transport, a number of laboratory-based tools have been developed to provide the much needed data. The most common approach is to use packed columns containing a porous geologic matrix of interest (e.g. sand or artificial media) designed to mimic soil or aquifer systems. In this approach, the concentration of nanoparticles eluting from the column outflow is monitored. Colloid filtration theory (and its variants) is then used to interpret the breakthrough curves and thus provide constants for predictive transport models (Choy et al. 2008; Tiraferri and Sethi 2009). While highly informative, the data obtained are a bulk average of a complex and heterogeneous array of interactions within the column. Even when intermediate sampling ports are inserted, they can only record bulk processes occurring between each sample port. These methods cannot unpick the impact that heterogeneities inherent in many natural systems, such as aquifers, soils, riverbeds and urban drainage systems have upon NP transport. These systems are often complex and display heterogeneous geometry, structure, hydrodynamics, geochemistry and microbiology. Breakthrough curves and invasive or destructive sampling of closed column experiments integrate colloid-media interactions over time or space, losing information relating to these complex environments. Even though the results are comparable, it masks many of the complexities that are related to controlling the overall rate of colloid transport. Moreover, even in homogeneous systems, changes in particle deposition can occur along the length of the column (Lecoanet and Wiesner 2004). This is significant as small changes in transport behaviour over the length of a packed column will make significant differences when scaled-up for field size applications. Consequently, there is a vital need to image inside packed columns to determine how spatial variability impacts our understanding of NP transport.

To overcome these issues, novel techniques have been developed which enable transport to be spatially resolved, including the use of fluorescent imaging protocols that visualize the transport of fluorescent particulates in translucent quartz matrices (Bridge et al. 2006). This, however, relies on sufficient photon penetration and thus uses translucent quartz and thin columns $(<1 \mathrm{~cm})$. The development of a scanning optical fibre fluorescence profiler has enabled the collection of some spatially resolved data inside opaque, packed columns (Shang et al. 2010). This system utilizes fluorescent nanoparticles and ten fibre optic detectors inserted at intervals into the column. While providing more spatial detail on NP transport, the 2D transport profile must still be inferred from the ten detector points (much of the column is still unseen). Gamma radiation and X-ray micortomography have been used for porous media characterization (IIlangasekare et al. 1995), fluid distribution (Brusseau et al. 2008) and solute-fluid transport (Hofstee et al. 1998) experiments. A major shortcoming of this method is at a single location; it requires relatively large counting times, leading to total counting times of several hours to produce an image (Werth et al. 2010). Also it would be difficult to resolve the smaller pore and fluid bodies especially the packed columns contains finer grains.

In this setting, magnetic resonance imaging (MRI) offers good potential to study particle transport patterns inside packed columns. In addition, it has previously been used to image particle and biofilm dynamics, reactive transport (Werth et al. 2010), sediment deposition (Heather et al. 2009), tracer transport through sediment bed (Lakshmanan et al. 2014), transport through finer grained system (Lakshmanan et al. 2015) and transport processes of heavy metals ions (Nestle et al. 2003). MR imaging can be used to collect high spatial resolution images of colloid and NP transport inside packed columns in two and three dimensions, when paramagnetically labelled particles are used (Baumann and Werth 2005; Ramanan et al. 2012). This technology works not by imaging the paramagnetic particle itself, but by imaging its effect on the $\mathrm{H}$ nuclei of surrounding water molecules. (Super) paramagnetic agents such as $\mathrm{Gd}^{3+}, \mathrm{Cu}^{2+} \mathrm{Mn}^{2+}$ and nanomagnetite cause a dramatic reduction in the relaxation time of adjacent $\mathrm{H}$ nuclei. This phenomenon can be used to quantitatively map the local concentration of paramagnetic nanoparticles within porous media, as there is a linear relationship between NP concentration and the change in water relaxation rates.

In a previous study, paramagnetic colloids $(1.3 \mu \mathrm{m})$ were imaged through a heterogeneous grain packing, each with a core of fine-grained silica gel surrounded by a shell of coarse-grained silica gel. The resultant spatially resolved data were scrutinized with CXTFIT and colloid filtration theory to reveal the impact of heterogeneity on transport (Baumann and Werth 2005). While most NP transport studies focus upon finer-grained systems of relevance to aquifers and soils. Here, however, we report the NP transport through coarser-grained systems which are relevant to riverbeds and the gravel matrices of sustainable urban drainage systems (SUDS). In this study, the transport of nanoparticles (50 $\mathrm{nm}$ in diameter) through packed columns of natural media (quartz and dolomite gravel) was imaged by MRI. We selected two gravel types: rose quartz and dolomite, as these exhibit different surface charge and thus would be expected to have different impacts on nanoparticle transport. Rose quartz has 
a zero charge between the $\mathrm{pH}$ of $2-3$, and it is negatively charged when the $\mathrm{pH}>3$ (Ibrahim et al. 2011). Dolomite has a zero charge at the $\mathrm{pH}$ of $8-8.1$ and exhibits a net positive charge below pH 8 (Pokrovsky et al. 1999). Rose quartz is negatively charged while dolomite exhibits a net positive charge at experimental $\mathrm{pH}$ conditions ( $\mathrm{Yu}$ et al. 2010)

Concentration profiles were analysed with CXTFIT and colloid filtration theory to estimate transport parameters at different time and distance intervals throughout the column. While in a previous study, we utilized MRI to examine the transport of two different nanomagnetitebased nanoparticles through quartz gravel (Ramanan et al. 2012), here we report on the transport of a single type of gadolinium-based NP through two different gravel types. Moreover, in the previous study, NP transport profiles at all time intervals along the column were fitted simultaneously to produce a single set of transport parameters. Here, we advance upon this by fitting the NP transport profiles at different time intervals individually, thus enabling us to examine the change in transport parameters with distance along the column. Mechanism of nanoparticle deposition and the interaction energy profiles was studied based on classic Derjaguin-Landau-Verwey-Overbeek (DLVO) theory.

This study was carried out from 2009 to 2011 at the School of Geographical and Earth Sciences and GEMRIC, University of Glasgow, Glasgow, UK.

\section{Materials and methods}

\section{Packed column}

Experiments were performed in saturated porous column containing randomly packed rose quartz and dolomite gravels with grain sizes of $1-5 \mathrm{~mm}$. The variation in grain shape and grain packing could contribute the heterogeneity in the column. The porosity of the column was determined from the weight of the column before and after saturation of the pore space with water. The porosity of rose quartz was 0.38 and dolomite was 0.46 . A cylindrical polypropylene column with the dimensions of $4.5 \mathrm{~cm}$ internal diameter and $9 \mathrm{~cm}$ height was used in this study. A porous sintered glass diffuser plate was placed on the inlet to provide an evenly distributed inflow of nanoparticle solution. The column was first filled with deionized water and then packed with rose quartz or dolomite grains in order to avoid air bubble formation. The commercially available paramagnetic nanoparticle Gado CELLTrack (referred to herein as $G d$ ) which exhibits a small net negative surface charge was used in this study (BioPAL, USA). The diameter of $50 \mathrm{~nm}$ (Loai et al. 2012) was used in the present study.
Transport experiments with Gd NP

The saturated packed column of rose quartz was placed vertically inside the horizontal bore of the MRI magnet, inside a 150-mm-diameter horizontal bird-cage RF coil inside the MRI. The flow was established using deionized water with a flow rate of $1 \mathrm{ml} / \mathrm{min}$ using a HPLC pump (Agilent 1100 Series). During the transport experiment, an inlet solution of $G d N P$ (at a concentration of $0.6 \mathrm{mM} \mathrm{Gd}$ ) was pumped into the packed column at a flow rate of $1 \mathrm{ml} /$ min, and the movement of nanoparticles through the system imaged every $5 \mathrm{~min}$. Each experiment was repeated in duplicate. The same procedure was repeated for dolomite gravel.

\section{Magnetic resonance imaging}

The MRI experiments were performed on a Bruker Avance BioSpec system, using a 30-cm horizontal bore, 7T superconducting magnet (Bruker BioSpin, Karlsruhe, Germany). A Bruker micro-imaging gradient insert (model BG-6), and 200-A gradient amplifiers were used to provide strong linear magnetic field gradient pulses of up to $1,000 \mathrm{mT} / \mathrm{m}$, thus allowing the system to perform microimaging experiments. Here, MRI was used to acquire spatially and temporally resolved $T_{2}$-weighted images of the column, while nanoparticles were transported through the column. Where $T_{2}$ is the spin-spin relaxation time of ${ }^{1} \mathrm{H}$ nuclei of the water phase. Further technical details of MR imaging can be found in Phoenix and Holmes 2008 and Callaghan 1991.

Nanoparticle transport imaging was performed using a Rapid Acquisition Relaxation Enhanced (RARE) Sequence. The experiments were performed with the following imaging parameter: echo time $\left(T_{E}\right) 170 \mathrm{~ms}$, repetition time $\left(T_{R}\right) 6,000 \mathrm{~ms}$ with a RARE factor of 48 , field of view was $9 \times 6 \mathrm{~cm}$, imaging matrix was $300 \times 192$ pixels, giving an in-plane resolution of $300 \times 312.5 \mu \mathrm{m}$ with a slice thickness of $1 \mathrm{~mm}$. To obtain comparable $T_{2}$ weighted images, these imaging parameters, including transmit and receiver gains, were kept identical during the entire experiment.

\section{Calibration of Gd NP concentrations}

The effect of nanoparticle concentration on the relaxation times of pure water is represented by the following equation (Bloembergen 1957; Phoenix and Holmes 2008)

$[C]=\frac{1}{R}\left[\frac{1}{T_{2, i}}-\frac{1}{T_{2,0}}\right]$

where $T_{2,0}$ is the relaxation time in the absence of paramagnetic NPs, $T_{2, i}$ is the relaxation time in the presence of 


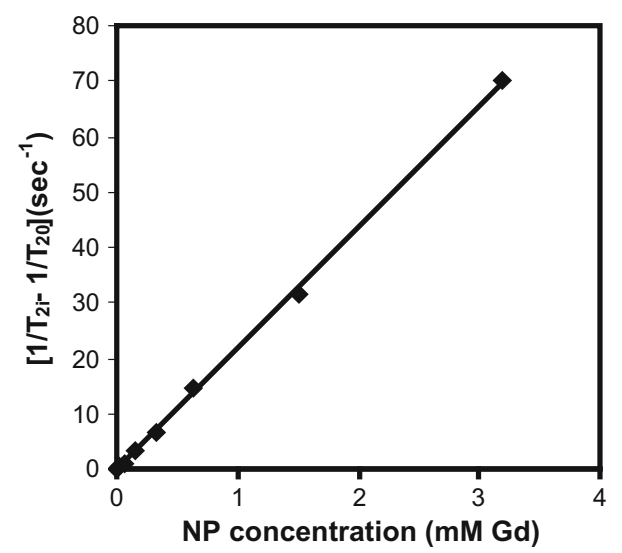

Fig. 1 Variation in the change of transverse relaxation rate with respect to $G d N P$ concentration (NP concentration shown as $\mathrm{mM}$ of Gd)

paramagnetic NPs, $[C]$ is the concentration of the paramagnetic NPs and $R$ is the relaxivity constant of the paramagnetic NPs. To determine the relaxivity constant $\mathrm{R}$, standards of different $G d N P$ concentrations were prepared and their $T_{2}$ values measured with a multiple spin-echo sequence with the following imaging parameters: echo time $\left(T_{E}\right) 9.2 \mathrm{~ms}$, repetition time $\left(T_{R}\right) \quad 10,000 \mathrm{~ms}$ and 200 echoes with two signal averages. A plot of $\left(1 / T_{2, i}-1 / T_{2,0}\right)$ versus NP concentration was shown in Fig. 1. The relaxivity constant, $\mathrm{R}$ (the slope of the line) was determined by the least square fit method to be $21.83 \mathrm{mM}^{-1} \mathrm{~s}^{-1}$.

\section{Quantitative measurement of Gd NP concentration}

The signal decay at a particular concentration $C$ is obtained using a RARE, and it is represented by (Haacke and al 1999);

$\frac{S}{S_{0}}=\exp \left(\frac{-t_{e}}{T_{2, i}}\right)$

where $S$ is the MRI signal, $S_{0}$ is the signal at zero echo time and $T_{2}$ is the spin-spin relaxation time. The effective echo time $t_{e}$ was chosen to maximize the signal contrast as described in von der Schulenburg et al. (2008) and was determined to be $170 \mathrm{~ms}$.

Combining Eqs. (1) and (2) gives the following equation:

$C=\left[\left(\ln \left(\frac{S}{S_{0}}\right) /-t_{e}\right)-\left(\frac{1}{T_{2,0}}\right)\right] \frac{1}{R}$

A RARE image of the sample containing only water was appropriately scaled to get a maximum signal intensity map of $S_{0}$. Hence, NP concentrations can be calculated from
Eq. (3), using $T_{2}$-weighted images acquired before and after introduction of NP.

By comparison of known inlet concentration with the inlet concentration determined from the calibration (Eq. 3), it was found that the calibration was offset by $-2 \%$ at the base (i.e. it gave slight negative values for zero), and the maximum concentration (measured at the inlet) was lower by $11 \%$. These artefacts were corrected by using a baseline correction factor and a stretching factor after the calibration.

\section{Transport parameters estimation}

Experimental results were compared to analytic solutions of the linear convection-dispersion equation (CDE) with a first-order loss term (Wraith and Or 1998):

$\frac{\partial}{\partial t} C=D \frac{\partial^{2} C}{\partial x^{2}}-v_{p} \frac{\partial C}{\partial x}-k C$

where $C$ is the concentration, $t$ is time, $x$ is distance from the inlet, $v_{p}$ average particle velocity, $D$ dispersion coefficient and $k$ is the decay coefficient. We assume a semi-infinite domain with constant concentration $C_{0}$ at the inlet.

$C(x, t)=C_{0}$ when $x=0, t \geq 0$

$C(x, t)=C_{0}$ when $0<x<\infty, t=0$

$\frac{d C(x, t)}{d x}=0$ when $x=\infty$

Equation (4) is linear, and an analytic solution exists for the boundary conditions in Eq. 5 (Toride and al 1995). Equation (4) is linear and hence Toride and al (1995) are able to use the principle of superposition to build up a solution for boundary conditions (5) by integrating impulse response functions. This solution is encoded in CXTFITExcel. The model parameters, $D, v_{p}$ and $k$ that give the best fit, measured by the least square error between modelled and observed concentration profiles were determined using CXTFIT-Excel computer package by Tang et al. (2010). Clearly, the model (Eq. 4) assumes constant parameter values and hence homogeneous characteristic dispersal, particle velocity and concentration-dependent losses. So, if the model holds then CXTFIT-Excel should yield the same $D, v p$ and $k$ from each fitting to each observed profile.

From the estimates of $k$ and the knowledge of the characteristics of the gravel, it is possible to gain further insights into the mechanism using colloidal filtration theory. Filtration theory is normally used to estimate the particle deposition. Under some limiting assumptions (Elimelech et al. 1995), the deposition rate can be equated to the decay rate in Eq. (4) and the determined form, 
$k=k^{\text {fast }} \alpha$

where $k^{\text {fast }}$ is the deposition rate that would occur if all particles that come into contact with grains stick, and $\alpha$ is the fraction of collisions that stick.From Baumann and Werth (2005), if the particles and grains are spherical,

$k^{\text {fast }}=\frac{3(1-\varepsilon)}{2 d_{10}} \eta v_{p}$

where $\varepsilon$ is the porosity, and $d_{10}$ is the grain diameter at the $10 \%$ mass fraction $\left(d_{10}\right.$ for dolomite is $4 \mathrm{~mm}$ and for rose quartz is $3.5 \mathrm{~mm}) . \eta$ is the frequency with which particles come into contact with grains and estimated by

$\eta=\frac{\left(\rho_{\mathrm{p}}-\rho_{\mathrm{f}}\right)}{18 \mu \nu} g d_{\mathrm{p}}^{2}+\frac{3}{2} A_{\mathrm{s}}\left(\frac{d_{\mathrm{p}}}{d_{\mathrm{g}}}\right)^{2}+0.897 \cdot \sqrt[3]{A_{\mathrm{s}}}\left(\frac{k_{\mathrm{B}} T}{\mu d_{\mathrm{p}} d_{\mathrm{g}} v}\right)^{\frac{2}{3}}$

where

$A_{\mathrm{s}}=\frac{1-\gamma^{5}}{1-1.5 \gamma+1.5 \gamma^{5}-\gamma^{6}}$,

and,

$\gamma=\sqrt[3]{1-\varepsilon}$

$d_{\mathrm{p}}$ and $d_{\mathrm{g}}$ denote the diameter of the particles and grains, $\rho_{\mathrm{p}}$ and $\rho_{\mathrm{f}}$ are the bulk density of the particle and the fluid, $\mu$ the fluid viscosity, $v$ pore water velocity, $g$ the gravitational acceleration, $A_{\mathrm{s}}$ a constant specific to the porous medium, $k_{\mathrm{B}}$ Boltzmann's constant, $T$ the temperature and $\varepsilon$ is the porosity.

\section{Results and discussion}

Transport of Gd NP through packed columns of rose quartz and dolomite

The transport of $G d N P$ into the packed column of rose quartz was recorded through a series of $T_{2}$-weighted images at 5-min time intervals. Selected time intervals are shown in Fig. 2. The presence of $G d N P$ shortens the $T_{2}$ values, and this is shown by the increase in measured MRI signal. Using the calibration protocol, these $T_{2}$-weighted images were converted into actual concentration maps of $G d N P$. An example $G d N P$ concentration map at $35 \mathrm{~min}$ is shown in Fig. 2(e).

The experiment was repeated, but with dolomite as the gravel matrix. The $T_{2}$-weighted images at various time intervals are shown in Fig. 3 . These $\mathrm{T}_{2}$-weighted images were converted into concentration maps of $G d N P$ using the calibration protocol, an example is shown in Fig. 3e.
All the data were obtained from a $2 \mathrm{D}$ slice through the centre of the column using rapid acquisition sequence. This is desirable for the interpretation of the data analysis and estimating the transport properties. Averaged NP concentrations across the width of the image slice at 5-min time intervals for rose quartz and dolomite column are shown in Fig. $4 \mathrm{a}$ and b. After $40 \mathrm{~min}$, the $G d N P$ starts to reach the outlet of the packed column in the rose quartz. For the dolomite, $G d N P$ took 65 min to reach the end of the column and thus is transported much more slowly than in rose quartz. Mass balance for the $G d N P$ was calculated, and it was observed that total $N P$ concentration from the concentration profiles was lower than that calculated from the pump rate; in dolomite, the recovery is $63 \%$ after $55 \mathrm{~min}$ and in rose quartz, the recovery is $84 \%$ after 25 min. From the concentration profiles, the total $N P$ concentration is lower for flow through dolomite column compared to rose quartz, and this is consistent with $G d N P$ attaching to grain surfaces.

As rose quartz and dolomite had slightly differing porosities ( 0.38 and 0.46 , respectively), and the transport profiles were normalized to pore volume for a more direct comparison of $N P$ progress (Fig. 5). This again highlighted the clearly slower transport through dolomite compared to rose quartz.

\section{Comparison of experimental data with CXTFIT analysis}

The experimental results from rose quartz and dolomite gravel were modelled with CXTFIT software. The transport parameters can be estimated at any point along the length of column. Here, we show four time intervals (20, 25, 30 and $35 \mathrm{~min}$ ) as an example (Fig. 6). Earlier time intervals are not shown as the number of data points representing the concentration profile are less and thus provide less reliable fits. (Figure 6). From the graph, it is evident that model profiles largely coinciding with the experimental data. The CXTFIT analysis achieved goodness-offit values $\left(R^{2}\right)$ from 95-99 \%. Further transport parameters derived from CXTFIT (dispersivity, $D, v_{p}$, and $k$ ) with $95 \%$ confidence interval are summarized in Table 1.

For dolomite gravel experiments, model profiles and the corresponding data for 40, 45, 50 and $55 \mathrm{~min}$ are shown in Fig. 7. These time intervals are chosen as they correspond to roughly the same distance along the column as the time intervals chosen for rose quartz. Similar to rose quartz experiments, the CXTFIT profiles largely coincide with the experimental data. The fitted transport parameters derived from CXTFIT (Dispersivity, $D, v_{p}$, and $k$ ) with $95 \%$ confidence intervals are summarized in Table 2. 

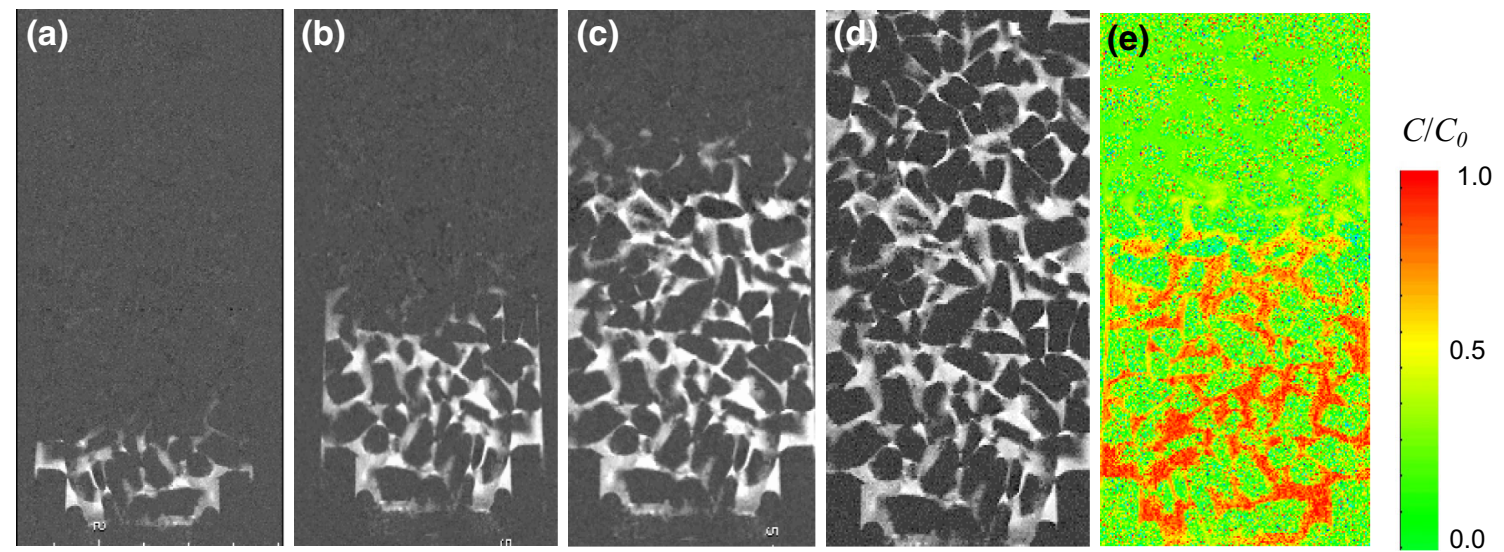

Fig. $2 T_{2}$-weighted images of $G d N P$ in quartz gravel at 10, 20, 40 and 45 min denoted by (a), (b), (c) and (d), respectively; NP transport is shown by bright regions. Figure $2 \mathrm{e}$ shows calibrated image of $G d N P$ concentration at $35 \mathrm{~min}$
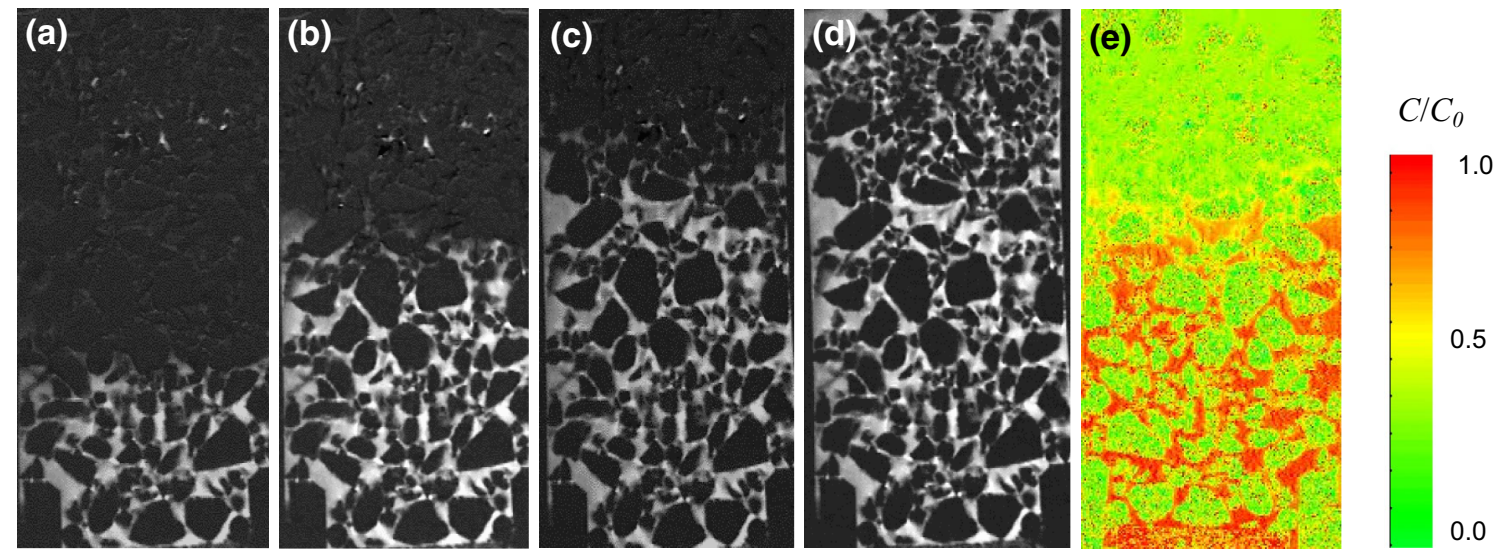

Fig. $3 T_{2}$-weighted images of $G d N P$ in dolomite gravel at 35, 45, 65 and 75 min denoted by (a), (b), (c) and (d), respectively; NP transport is shown by bright regions. Figure $3 \mathbf{e}$ shows calibrated image of $G d N P$ concentration at $55 \mathrm{~min}$

The averaged dispersivity for rose quartz was $5.2 \times 10^{-3}$ and $3.28 \times 10^{-3} \mathrm{~m}$ for dolomite gravel. Ramanan et al. (2012) studied NP transport through similar quartz gravel and reported a dispersivity of $3.5 \times$ $10^{-3} \mathrm{~m}$ which is comparable to our results. Averaged dispersion coefficient, $D_{\text {ave }}$ for rose quartz in this study were $1.92 \times 10^{-8} \mathrm{~m}^{2} / \mathrm{s}$ and for dolomite gravel $1.43 \times$ $10^{-8} \mathrm{~m}^{2} / \mathrm{s}$. Khrapitchev and Callaghan (2003) reported the dispersive flow in porous media for various literature studies. Their relationship gives a $D_{\text {ave }}$ value of $6.40 \times$ $10^{-8} \mathrm{~m}^{2} / \mathrm{s}$ for rose quartz and $7.20 \times 10^{-8} \mathrm{~m} 2 / \mathrm{s}$ for dolomite gravel which are in the same order of magnitude. While the determined values appear appropriate, thus supporting the robustness of the MRI approach, it is evident both dispersivity and $D$ were not constant but increased over the analysed range by a factor of 3 and 4 for rose quartz and dolomite, respectively, (Table 1, 2). As time represents the progress of the NP plume, changes that occur with time must also be considered changes that occur with distance. Although the time intervals chosen for the two gravel types were different, these correspond to roughly the same distances along the column (front of plume at approximately 4, 5.2, 6.4 and $7.2 \mathrm{~cm}$ ). The other CXTFIT derived parameters also showed variability; $v_{p}$ increased by a factor of $\sim 2$ in both gravels over the time and distance analysed, while $k$ decreased by a factor of 1.6 and 2.4 in quartz and dolomite, respectively (Table 1, 2). The fast deposition rate constant $k^{\text {fast }}$ and collision efficiency $\alpha$ were then calculated using Eqs. 6 and 7 .

Estimation of DLVO interaction energy profiles

Particle deposition behaviour in saturated porous media for colloids and nanoparticles is reported in the literature 

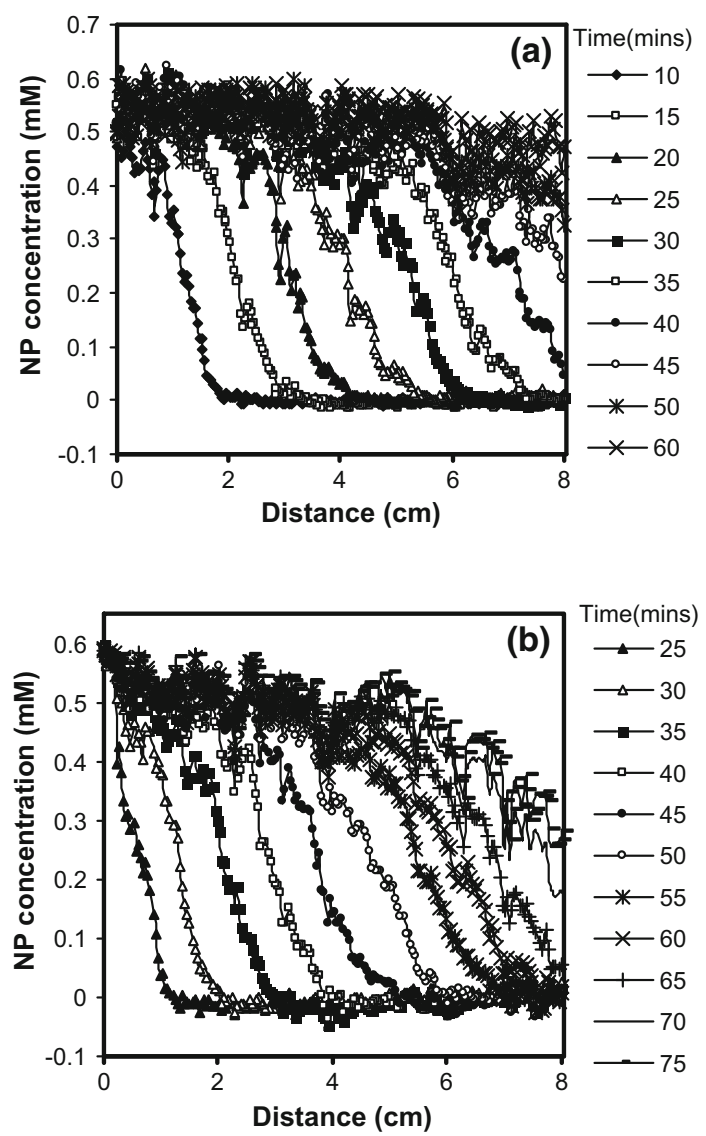

Fig. 4 Averaged concentration profiles of $G d N P$ concentration (reported as $\mathrm{mM} \mathrm{Gd}$ ) at various time intervals a rose quartz, b dolomite column experiments

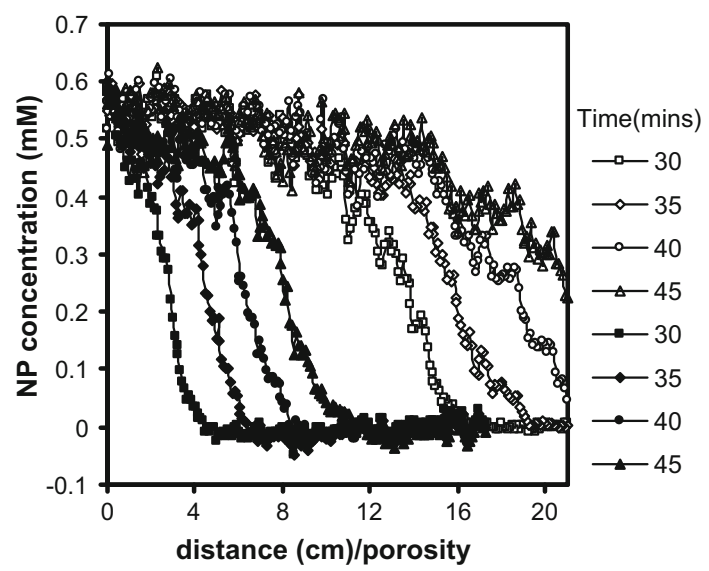

Fig. $5 G d N P$ concentration at various time intervals for rose quartz (open symbol) and dolomite (closed symbol), normalized to porosity

(Tufenkji and Elimelech 2005 and Ramanan et al. 2012). Elimelech et al. (1990) classified particle deposition as favourable (absence of repulsive interaction energies) and unfavourable (predominate repulsive colloidal interactions). When the particle and collector have similar charges, deposition can occur in secondary energy minima, and it is smaller for NP than colloids.

Derjaguin-Landau-Verwey-Overbeek theory (Derjaguin and Landau 1941; Verwey 1948) is applied to estimate the interaction energy profiles. The total interaction potential is described as the sum of electrostatic repulsion $\left(V_{\text {rep }}\right)$ and the attractive potential $\left(V_{\text {att }}\right)$. This was determined for the NP-rose quartz and NP-dolomite systems by treating as a sphere-plate interaction. $V_{\text {rep }}$ and $V_{\text {att }}$ were calculated using the expression of Hogg et al. (1966) and Gregory (1981). Zeta potential values for rose quartz and dolomite gravel were taken from the literature (Jada et al. 2006; Marouf et al. 2009). Hamaker constant of $10^{-20} J$ was used in this analysis (Elimelech and O'Meilat 1990; Chowdhury et al. 2011).

DLVO interaction energy profiles for NP-rose quartz and dolomite are shown in Fig. 8a and b. It reveals the presence of repulsive energy barrier in NP-rose quartz (unfavourable condition due to the negatively charged surfaces) with secondary energy minima. An electrostatically favourable condition was observed in NP-dolomite system, and this type of deposition occurs in primary energy minimum.

In Fig. 8c, the total interaction energy profiles are replotted on a different scale; the height of energy barrier $12 k T$ and the depth of the secondary energy minimum of $0.78 \mathrm{kT}$ were measured in NP-rose quartz system, and no energy barrier was observed in NP-dolomite system. So, similarly charged surfaces should have a more recovery than the other one, and this was observed in our mass balance calculations.

\section{Mechanisms of NP deposition with DLVO}

In the present study, the average collision efficiency $\alpha$ was higher in dolomite than rose quartz, consistent with an attractive electrostatic force between the negatively charged NP and positively charged dolomite gravel, which thus displays greater retardation. In contrast, there is less attraction (or greater repulsion) between the negatively charged NP and the negative rose quartz, which thus displays less retardation. However, despite little electrostatic attraction, retardation in the rose quartz does occur, likely due to deposition of NPs in weak secondary energy minima (Petosa et al. 2010; Hahn et al. 2004) which we also observed in the DLVO energy profiles. This type of deposition is considered reversible. This reversible deposition has direct implications in colloidal transport and particle deposition kinetics (Tufenkji and Elimelech 2005). 
Fig. $6 G d N P$ concentration profile of rose quartz experiment at a $20 \mathrm{~min}$, b $25 \mathrm{~min}$, c $30 \mathrm{~min}, \mathbf{d} 35 \mathrm{~min}$. Experimental data (closed symbol) and CXTFIT data (open symbol)
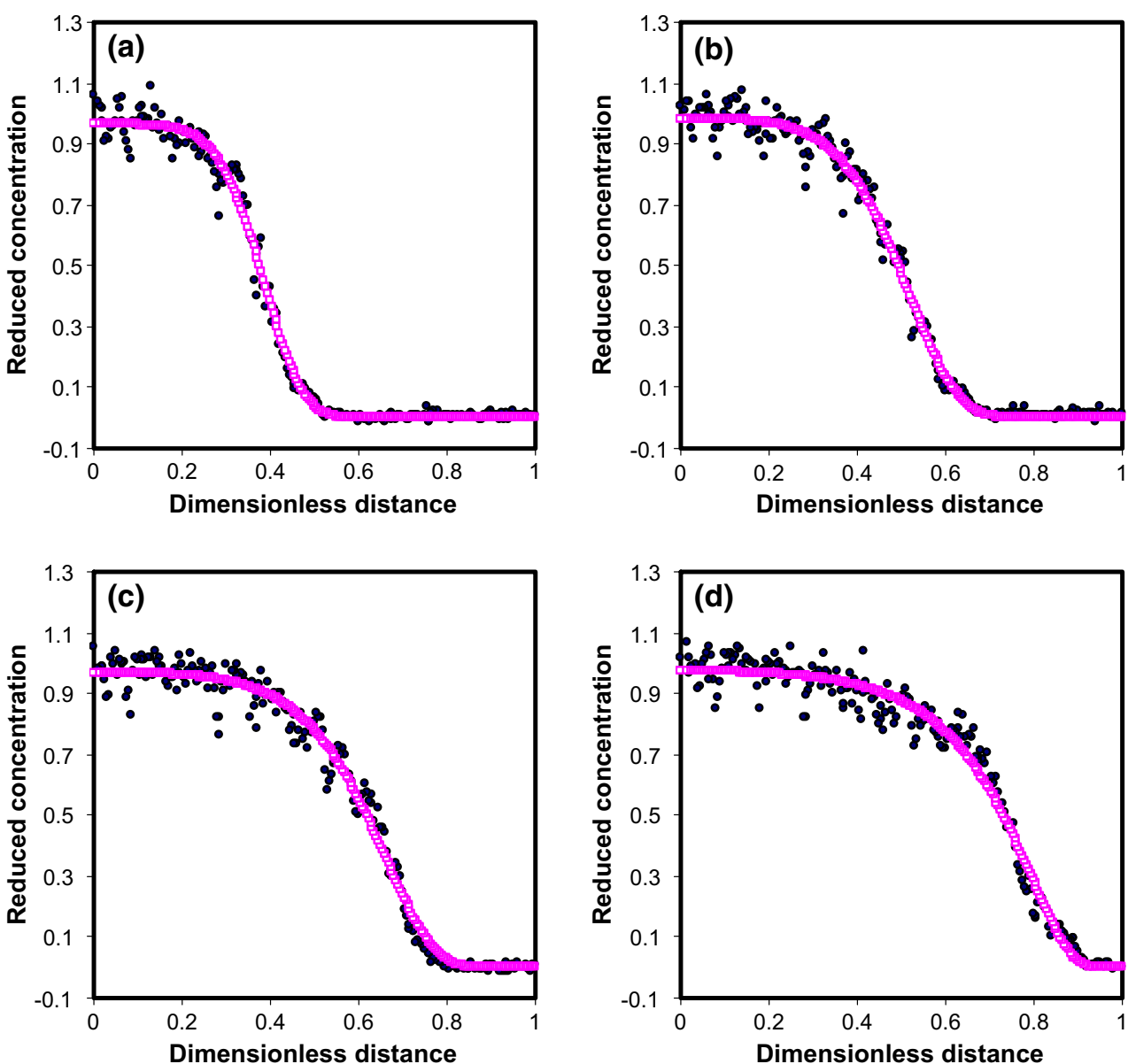

In contrast, the strong electrostatic attraction between the NP and dolomite likely results in NP deposition in primary energy minima, ensuring significant and permanent attachment of NPs to the grain surface (Darlington et al. 2009, Petosa et al. 2010). Examination of variation in $\alpha$ over time and distance for the two gravels revealed this parameter decreased by a factor of 3.3 and 4.5 for rose quartz and dolomite, respectively (Table 1, 2).

The observed increase in dispersivity and decrease in collision efficiency over time may indicate surface charge heterogeneities on the NPs, and the reduced availability of attachment sites on the collector surface can control the particle deposition (Tufenkji and Elimelech 2005 and Chowdhury et al. 2011). These factors prompt a fraction of the most favourable NPs to attach to grain surfaces more readily early on. In the case of dolomite, the most favourably attaching NPs would be those of greatest negative surface charge, while for rose quartz, attachment would favour those with the weakest negative surface charge. Those that are unattached are transported forward,
Table 1 Estimated transport parameters using CXTFIT for rose quartz

\begin{tabular}{lllll}
\hline \multirow{2}{*}{$\begin{array}{l}\text { Transport } \\
\text { parameters }\end{array}$} & \multicolumn{4}{l}{ Various time intervals (mins) } \\
\cline { 2 - 5 } & 20 & 25 & 30 & 35 \\
\hline $\begin{array}{c}\text { Dispersivity } \\
(\mathrm{m})\end{array}$ & $2.5 \times 10^{-3}$ & $4.2 \times 10^{-3}$ & $6.9 \times 10^{-3}$ & $7.5 \times 10^{-3}$ \\
$\mathrm{D}\left(\mathrm{m}^{2} / \mathrm{s}\right)$ & $9.12 \times 10^{-9}$ & $1.525 \times 10^{-8}$ & $2.501 \times 10^{-8}$ & $2.73 \times 10^{-8}$ \\
$v_{p}(\mathrm{~m} / \mathrm{s})$ & $3.97 \times 10^{-5}$ & $4.8 \times 10^{-5}$ & $6.2 \times 10^{-5}$ & $8.17 \times 10^{-5}$ \\
$k(1 / \mathrm{s})$ & $6.58 \times 10^{-5}$ & $5.76 \times 10^{-5}$ & $4.94 \times 10^{-5}$ & $4.11 \times 10^{-5}$ \\
$k^{\text {fast }}(1 / \mathrm{s})$ & $3.39 \times 10^{-4}$ & $4.1 \times 10^{-4}$ & $6.38 \times 10^{-4}$ & $6.99 \times 10^{-4}$ \\
$\begin{array}{c}\text { Collision } \\
\text { efficiency, }\end{array}$ & 0.194 & 0.141 & 0.077 & 0.058 \\
$\alpha$ & & & & \\
\hline
\end{tabular}

but bind to collector surfaces less readily. Blocking occurs when previously deposited particles hinder subsequent deposition and thus attachment decreases with distance. For both gravels, changes in dispersivity and $\alpha$ would not be detectable from standard breakthrough curve analysis of 
Fig. $7 G d N P$ concentration profile of dolomite gravel experiment at a $40 \mathrm{~min}$, b $45 \mathrm{~min}$, c $50 \mathrm{~min}$, d $55 \mathrm{~min}$. Experimental data (closed symbol) and CXTFIT data (open symbol)
Table 2 Estimated transport parameters using CXTFIT for dolomite gravel
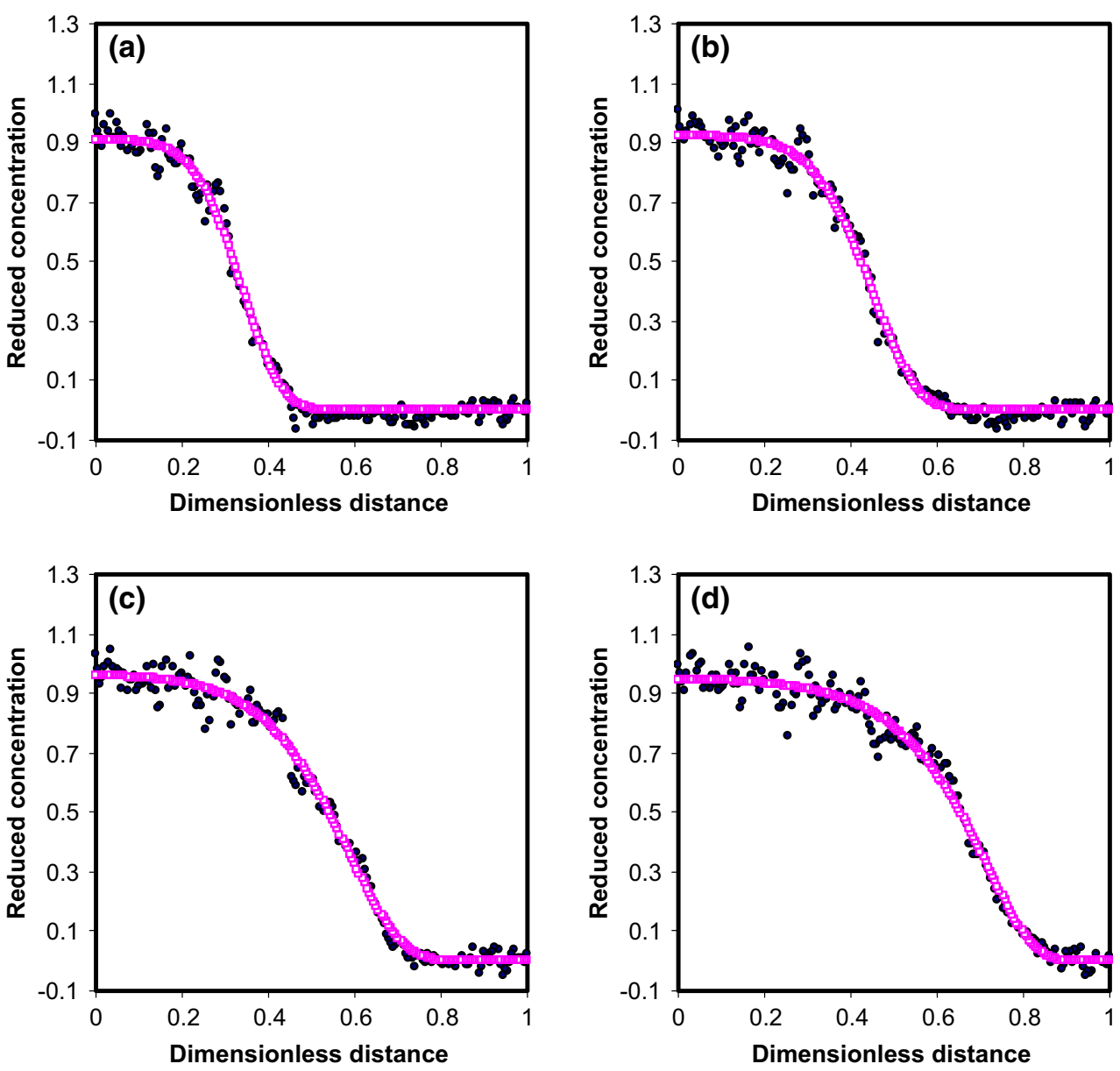

\begin{tabular}{lllll}
\hline Transport parameters & \multicolumn{4}{l}{ Various time intervals (mins) } \\
\cline { 2 - 5 } & 40 & 45 & 50 & 55 \\
\hline Dispersivity $(\mathrm{m})$ & $1.2 \times 10^{-3}$ & $2.4 \times 10^{-3}$ & $4.5 \times 10^{-3}$ & $5.1 \times 10^{-3}$ \\
$\mathrm{D}\left(\mathrm{m}^{2} / \mathrm{s}\right)$ & $5.078 \times 10^{-9}$ & $1.504 \times 10^{-8}$ & $1.987 \times 10^{-8}$ & $2.208 \times 10^{-8}$ \\
$v_{p}(\mathrm{~m} / \mathrm{s})$ & $3.71 \times 10^{-5}$ & $4.35 \times 10^{-5}$ & $5.27 \times 10^{-5}$ & $6.76 \times 10^{-5}$ \\
$k(1 / \mathrm{s})$ & $6.79 \times 10^{-5}$ & $6.11 \times 10^{-5}$ & $3.4 \times 10^{-5}$ & $2.72 \times 10^{-5}$ \\
$k^{\text {fast }}(1 / \mathrm{s})$ & $2.11 \times 10^{-4}$ & $2.48 \times 10^{-4}$ & $3.0 \times 10^{-4}$ & $3.85 \times 10^{-4}$ \\
Collision efficiency, $\alpha$ & 0.322 & 0.247 & 0.113 & 0.071 \\
\hline
\end{tabular}

NP concentrations eluting from the end of the column. Invariably, incorrect transport parameters would be determined. However, with MRI, these variations can be observed.

Transport in such a coarse grain system has relevance to riverbeds and the gravel matrices of SUDS. NPs are released into these aquatic environments during manufacture, transport and disposal. The variations in transport parameters seen along the length of the experimental column are significant when considered for field scale application. A lack of understanding of whether, and how, transport parameters change over the length of the column could lead to the determination of incorrect transport parameters. Deviations from true values would be magnified when applied to predict NP transport overmuch larger distance at field scale in systems such as SUDS or riverbeds. Consequently, it is imperative to be able to understand how transport phenomena vary with distance. 

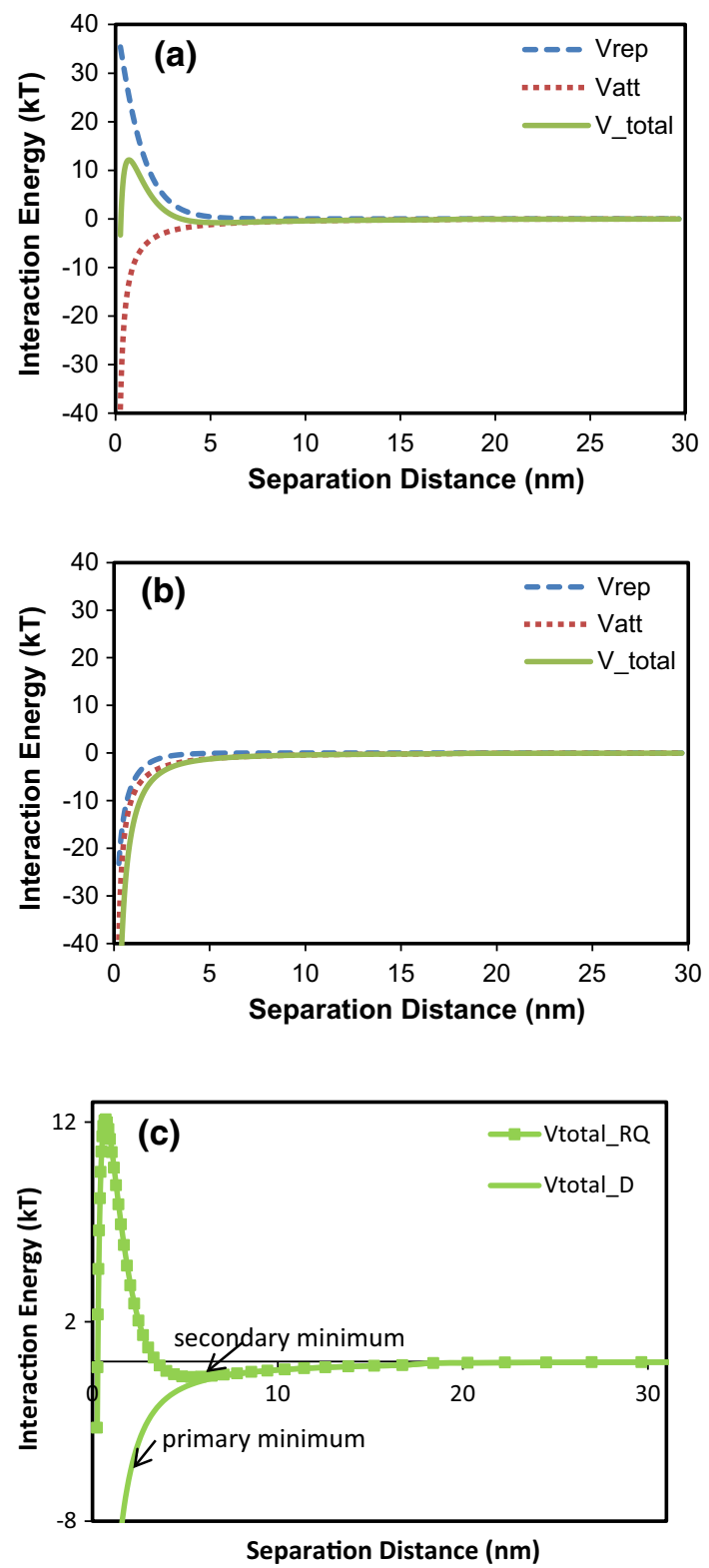

Fig. 8 Interaction energy profile generated with DLVO theory for a NP-RQ, b NP-D, c Total energy replots for RQ and D

Moreover, natural and engineered gravel systems such as river beds and SUDS can exhibit significant heterogeneity in geometry, structure, hydrodynamics, geochemistry and microbiology throughout. These will undoubtedly generate further complexities in NP transport which will be difficult to unpick with standard breakthrough curves. These complexities would again require the type of spatially resolved data collection that MRI can provide.

The current system has a vertical column setup and could be modified into a horizontal arrangement which is similar to river bed. Flowing with NP over the top of the gravel matrix would enable to study the NP transport in riverbeds. Also NP transport of interactions with the grain surfaces could be explored further with the impact of NPs parameters such as a shape, molecular mass and surface charge upon their transport within the system.

\section{Conclusion}

The transport of paramagnetic NPs in two coarse-grained porous column made up of positively charged dolomite and negatively charged rose quartz gravel grains was studied using MRI. NP transport of interactions with the grain surfaces were successfully imaged temporally and in two dimensions with MRI. The spatially and temporally resolved data were analysed using CXTFIT software with goodness-of-fit values $\left(R^{2}\right)$ from 95 to $99 \%$. The results reported here indicated that key transport parameters such as dispersivity and the collision efficiency varied along the length of the column.

From the estimated DLVO interaction energy profiles, electrostatically favourable condition was observed in NPdolomite system, and the presence of repulsive energy barrier was observed in NP-rose quartz. The deposition mechanism was discussed along with the measured transport parameters. The average collision efficiency $\alpha$ was higher in dolomite than rose quartz, and this is consistent with the strong electrostatic attraction between the NP and dolomite. These variations would not be detectable by standard breakthrough curve analysis on NPs eluting from the end of the column. An alternative approach, the present study illustrates the ability of MRI to non-invasively image NP transport inside porous media. Transport in such a coarse grain system has relevance to riverbeds and the gravel matrices of SUDS.

Acknowledgments This work was supported by grants from the Engineering and Physical Sciences Research Council (EP/G028443/ 1) and Natural Environmental Research Council (NE/G010269/1).We also thank Jim Mullen for his assistance with MRI experiments.

\section{References}

Baumann T, Werth CJ (2005) Visualization of colloid transport through heterogeneous porous media using magnetic resonance imaging. Colloids Surf A Physicochem Eng Asp 265:2-10

Bloembergen N (1957) Proton relaxation times in paramagnetic solutions. J Chem Phys 27:572-573 
Boxall AB, Tiede K, Chaudhry Q (2010) Engineered nanomaterials in soils and water: How do they behave and could they pose a risk to human health? Crit Rev Toxicol 40(7):653-670

Bridge JW, Banwart SA et al (2006) Noninvasive quantitative measurement of colloid transport in mesoscale porous media using time lapse fluorescence imaging. Environ Sci Technol 40:5930-5936

Brusseau ML, Janousek H et al (2008) Synchrotron X-ray microtomography and interfacial partitioning tracer test measurements of NAPL-water interfacial areas. Water Resour Res 44:1-9

Callaghan PT (1991) Principles of nuclear magnetic resonance microscopy. Oxford Science Publications, Oxford

Chowdhury I, Hong Y et al (2011) Mechanisms of TiO2 nanoparticle transport in porous media: role of solution chemistry, nanoparticle concentration, and flow rate. J Colloid Interface Sci 360:548-555

Choy CC, Wazne M et al (2008) Application of an empirical transport model to simulate retention of nanocrystalline titanium dioxide in sand columns. Chemosphere 71:1794-1801

Darlington TK, Neigh AM et al (2009) Nanoparticle characteristics affecting environmental fate and transport through soil. Environ Toxicol Chem 28:1191-1199

Derjaguin BV, Landau LD (1941) Theory of the stability of strongly charged lyophobic sols and of the adhesion of strongly charged particles in solutions of electrolytes. Acta Physicochim. USSR 14:733-762

Elimelech M, O'Meilat CR (1990) Kinetics of deposition of colloidal particles in porous media. Environ Sci Technol 24:1528-1536

Elimelech M, Gregory J et al (1995) Particle deposition and aggregation. Measurement, modelling and simulation. Butterworth-Heinemann Ltd, Jordan Hill, Oxford

Gregory J (1981) Approximate expressions for retarded van der Waals interaction. Colloid Interface Sci 83:138-145

Haacke EM, Brown RW et al (1999) Magnetic resonance imaging: physical principles and sequence design. Wiley, Liss

Hahn M, Abadzic D et al (2004) Aquasols: on the role of secondary minima. Environ Sci Technol 38:5915-5924

Handy RD, Kammer FVD et al (2008) The ecotoxicology and chemistry of manufactured nanoparticles. Ecotoxicology 17:287-314

Heather H, Elisa V, William H (2009) Using magnetic resonance imaging for experimental analysis of fine-sediment infiltration into gravel beds. Sedimentology 56:1961-1975

Hofstee C, Oostrom M et al (1998) Infiltration and redistribution of perchloroethylene in partially saturated stratified porous media. J Contam Hydrol 34:293-313

Hogg R, Healy TW, Fuerstenau DW (1966) Mutual coagulation of colloidal dispersions. Trans Faraday Soc 62:1638-1652

Ibrahim I, Hussin $\mathrm{H}$ et al (2011) A study on the interaction of feldspar and quartz with mixed anionic/cationic collector. J Fundam Sci 7:101-107

Illangasekare TH, Ramsey JL et al (1995) Experimental study of movement and distribution of dense organic contaminants in heterogeneous aquifers. J Contam Hydrol 20:1-25

Jada A, Ait Akbour R, Douch J (2006) Surface charge and adsorption from water onto quartz sand of humic acid. Chemosphere 64:1287-1295

Khrapitchev AA, Callaghan PT (2003) Reversible and irreversible dispersion in a porous medium. Phys Fluids 15(9):2649-2660

Lakshmanan S, Gareth P, Heather H, William MH (2014) Characterization of flow transport within pore spaces of an open-work gravel bed. Int J Eng Technol 3:457-463
Lakshmanan S, William MH, William TS, Vernon RP (2015) Nanoparticle transport in saturated porous medium using magnetic resonance imaging. Chem Eng J 266:156-162

Lecoanet HF, Wiesner MR (2004) Velocity effects on fullerene and oxide nanoparticle deposition in porous media. Environ Sci Technol 38:4377-4382

Loai Y, Ganesh T, Cheng HLM (2012) Concurrent dual contrast for cellular magnetic resonance imaging using gadolinium oxide and iron oxide nanoparticles. Int J Mol Imaging 2012:1-10

Marouf R, Marouf-Khelifa K et al (2009) Zeta potential study of thermally treated dolomite samples in electrolyte. Microporous Mesoporous Mater 122:99-104

Nel A, Xia T, Mädler L, Li N (2006) Toxic potential of materials at the nanolevel. Science 311:622-627

Nel A, Xia T, Meng H, Zhang H (2013) Nanomaterial toxicity testing in the 21st century: use of a predictive toxicological approach and high throughput screening. Acc Chem Res 46(3):607-621

Nestle N, Wunderlich A et al (2003) Spatial and temporal observations of adsorption and remobilization of heavy metal ions in a sandy aquifer matrix using magnetic resonance imaging. Environ Sci Technol 37:3972-3977

Petosa A, Jaisi DP et al (2010) Aggregation and deposition of engineered nanomaterials in aquatic environments: role of physicochemical interactions. Environ Sci Technol 44:6532-8549

Phoenix VR, Holmes WM (2008) Magnetic resonance imaging of structure, diffusivity and copper immobilization in a phototrophic biofilm. Appl Environ Microbiol 74:4934-4943

Pokrovsky OS, Schott J, Thomas F (1999) Dolomite surface speciation and reactivity in aquatic systems. Geochim Cosmochim Acta 63:3133-3143

Ramanan B, Holmes WM et al (2012) Investigation of nanoparticle transport inside coarse-grained geological media using magnetic resonance imaging. Environ Sci Technol 46:360-366

Scown TM, van Aerle R, Tyler CR (2007) Review: do engineered nanoparticles pose a significant threat to the aquatic environment. Nanomedicine 2(6):919-927 Lond

Shang J, Liu C et al (2010) In-situ measurements of engineered nanoporous particle transport in saturated porous media. Environ Sci Technol 44:8190-8195

Tang G, Mayes MA et al (2010) CXTFIT/Excel-A modular adaptable code for parameter estimation, sensitivity analysis and uncertainty analysis for laboratory or field tracer experiments. Comput Geosci 136:1200-1209

Tellam JH (2011) Manufactured nanoparticles: a new threat to the security of some groundwater supplies? Clim Chang Eff Water Resour NATO Sci Peace Secur Ser C: Environ Secur 3:139-146

Tiraferri A, Sethi R (2009) Enhanced transport of zerovalent iron nanoparticles in saturated porous media by guar gum. J Nanopart Res 11:635-645

Toride N, Leij FJ et al (1995) The CXTFIT code for estimating transport parameters from laboratory or field tracer experiments, version 2.0, research report no. 137. US Salinity Laboratory, USDA, ARS, Riverside

Tufenkji N, Elimelech M (2005) Breakdown of colloid filtration theory: role of the secondary energy minimum and surface charge heterogeneities. langmuir 21:841-852

Verwey EJW, Overbeek JThG (1948) Theory of the stability of lyophobic colloids. Elsevier, Amsterdam

Von der Schulenburg DAG, Holland DJ et al (2008) Spatially resolved quantification of metal ion oncentration in a biofilmmediated ion exchanger. Biotechnol Bioeng 99(4):821-829 
Werth CJ, Zhang C et al (2010) Nanoparticles: their potential toxicity, waste and environmental management. J Contam Hydrol 113:1-24

Wraith JM, Or D (1998) Non linear parameter estimation using spreadsheet software. J Nat Resour Life Sci Educ 27:13-19
Yu J, Berlin JM et al (2010) Transport study of nanoparticles for oilfield application. SPE Int Conf Oilfield Scale 10:512-528 Aberdeen, UK 